\title{
ON THE EXTENSION OF THE ZAK TRANSFORM
}

\author{
Ahmed I. Zayed and Piotr Mikusiński
}

\begin{abstract}
The Zak transform, which is a unitary transformation of $L^{2}(\Re)$ onto $L^{2}(Q)$ where $Q$ is the unit square, has been extended to larger spaces of functions and generalized functions by many people, including A. J. E. M. Janssen.

In this paper, we present a new extension of the Zak transform. The novelty of our approach is that it extends the Zak transform by using its algebraic and convolution structure instead of using its analytic properties, which are not easy to deal with. Our extension produces a topological isomorphism between the domain and the range of the transform. Moreover, it enables us to use the Zak transform in situations where other extensions do not apply.
\end{abstract}

\section{Introduction}

In 1967, J. Zak introduced a transform, now known as the Zak transform, to construct a quantum mechanical representation for the description of the motion of a Bloch electron in the presence of a magnetic or electric field [11]. He called it the $k$ $q$ transform and also used it to study the motion of an electron in a periodic potential and a constant magnetic field. Interest in this transform has been revived in recent years because of its relationship to different types of coherent states, one of which is the affine coherent states, commonly known nowadays as wavelets $[2,4]$. Another type of coherent states that is more intimately related to the Zak transform is the Weyl-Heisenberg states, which is a family of functions of the form $\left\{g_{m b, n a}(x)=e^{2 \pi i m b} g(x-n a)\right\}_{m, n \in Z}$, where $g \in L^{2}(\Re), Z$ is the set of integers, and $a, b$ are real numbers. Since $m, n \in Z$, there is no loss of generality in restricting $a$ and $b$ to positive numbers.

A question of great importance in both physics and mathematics is this: under what conditions on $g, a$, and $b$ does the family $\left\{g_{m b, n a}\right\}_{m, n \in Z}$, form a frame or an orthonormal basis of $L^{2}(\Re)$ ?

After several years of intensive research, the answer is now well understood.

1) If $a b>1$, there is no $g \in L^{2}(\Re)$ for which $\left\{g_{m b, n a}\right\}_{m, n \in Z}$, is a frame.

2) If $a b<1$ and $g$ is chosen appropriately, then the family $\left\{g_{m b, n a}\right\}_{m, n \in Z}$ can be a frame and even more, it can be a tight frame with good time-frequency localization, yet it cannot be an orthonormal basis.

3) In the critical case when $a b=1,\left\{g_{m b, n a}\right\}_{m, n \in Z}$ can be a frame for some suitable function $g$; however, the frame will have bad time-frequency localization in the sense that either $x g(x) \notin L^{2}(\Re)$ or $\omega \widehat{g}(\omega) \notin L^{2}(\Re)$, where $\widehat{g}$ is the Fourier transform of $g$.

Received June 10, 1994, revised October 5, 1994.

1991 Mathematics Subject Classification. Primary 42A38, 44A15, Secondary 46F12, 44A40.

Key words and phrases: Zak transform, Boehmian, generalized functions.

This research was supported by a grant from the Department of Sponsored Research at the University of Central Florida, Grant \#9043j. 
In contrast with the other two cases, in the critical case and only in this case can the family $\left\{g_{m b, n a}\right\}_{m, n \in Z}$ be an orthonormal basis. The Zak transform has played a vital role in proving the results in this critical case; see [4] and [2], p. 110. In addition to that, the Zak transform has been used directly and indirectly in solving different physics and engineering problems, in particular, in solid state physics and signal analysis. We refer the reader to [5] and [6] for references on some of the applications of the Zak transform and its development.

The Zak transform maps a function of one variable into a function of two variables. In fact, it is a unitary transformation from $L^{2}(\Re)$ into $L^{2}(Q)$, where $Q=[0,1] \times[0,1]$ is the unit square in the time-frequency plane. Since its introduction in 1967, the Zak transform has been studied by several people, including A. Janssen $[5,6]$, who developed some of its properties and extended it to function and generalized function spaces that are more general than $L^{2}(\Re)$.

Borrowing some of V. Bargmann's ideas regarding the Bargmann transform, Janssen [5] was able to extend the Zak transform to the space $\left(S_{1 / 2}^{1 / 2}\right)^{*}$, the dual space of the space $S_{1 / 2}^{1 / 2}$. The latter is a special case of the space $S_{\alpha}^{\beta}$ introduced by Gel'fand and Shilov in [3]. Janssen's approach utilized the analytic properties of both the Zak transform and the space $S_{1 / 2}^{1 / 2}$.

In this paper, we extend the Zak transform to a space of generalized functions that is an algebraic extension of $L^{2}(\Re)$. The novelty of our approach is that it uses the algebraic rather than the analytic properties of the Zak transform, and hence it offers a more elegant approach to the extension problem. Moreover, it also sheds some light on less known properties of the Zak transform that turn out to be an important tool in our extension. The new extension of the Zak transform is applied to some generalized functions that are not included in other known extensions.

\section{The Zak transform}

In this section, we introduce the Zak transform and some of its properties that will be needed in the sequel.

The Zak transform of a function $f$ is formally defined by

$$
(Z f)(t, \omega)=\sqrt{a} \sum_{k \in Z} f(t+k a) e^{-2 \pi i k a \omega},
$$

where $t, \omega \in \Re$ and $a$ is a fixed parameter greater than zero.

It is well defined if, for example, $f$ is continuous and has compact support or if $f(x)=O\left(1 /(1+|x|)^{1+\varepsilon}\right)$ as $|x| \rightarrow \infty$ for some $\varepsilon>0$. For a fixed $t$, the Zak transform can be viewed formally as the discrete Fourier transform of the sequence $\{f(t+k a)\}_{k \in Z}$. It is readily seen that

$$
\begin{gathered}
(Z f)\left(t, \omega+\frac{1}{a}\right)=(Z f)(t, \omega), \\
(Z f)(t+a, \omega)=e^{2 \pi i a \omega}(Z f)(t, \omega) ;
\end{gathered}
$$

thus, the Zak transform maps a function of one variable into a function of two variables that is completely determined by its values on the rectangle $[0, a] \times[0,1 / a]$ in the timefrequency plane, i.e., $t-\omega$ plane. 
To simplify the notation, we shall take $a=1$; however, it should be emphasized that all the results we are going to derive in this article can be obtained for any $a>0$, by introducing an appropriate scaling factor. Thus, we have

$$
\begin{gathered}
(Z f)(t, \omega)=\sum_{k \in Z} f(t+k) e^{-2 \pi i k \omega}, \quad t, \omega \in \Re, \\
(Z f)(t, \omega+1)=(Z f)(t, \omega), \\
(Z f)(t+1, \omega)=e^{2 \pi i \omega}(Z f)(t, \omega) .
\end{gathered}
$$

We define the translation and modulation operators $T_{a}$ and $E_{b}$, respectively, by

$$
T_{a} f(x)=f(x-a), \quad E_{b} f(x)=e^{-2 \pi i b x} f(x) .
$$

When $f=1$, we write $E_{b} 1(x)=e^{-2 \pi i b x}=E_{b}(x)$. The Fourier transform of a function $f(x)$ is formally defined by

$$
\widehat{f}(\omega)=F(\omega)=\int_{-\infty}^{\infty} f(x) e^{2 \pi i \omega x} d x
$$

so that the inverse transform is given by

$$
f(x)=\int_{-\infty}^{\infty} \widehat{f}(\omega) e^{-2 \pi i x \omega} d \omega=(\widehat{f}(w))^{\vee}
$$

with $(\cdot)^{\vee}$ being defined by

$$
\stackrel{\vee}{g}(x)=\int_{-\infty}^{\infty} g(\omega) e^{-2 \pi i x \omega} d \omega .
$$

Using the standard inner product on $L^{2}(\Re)$,

$$
\langle f, g\rangle=\int_{-\infty}^{\infty} f(x) \overline{g(x)} d x
$$

we have

$$
\widehat{f}(\omega)=\left\langle f, E_{\omega}\right\rangle \text { and } f(x)=\left\langle\widehat{f}, \bar{E}_{x}\right\rangle,
$$

where $\bar{f}$ denotes the complex conjugate of $f$.

For $m$ and $n$ integers, it is easy to verify that

$$
Z\left(E_{m} T_{n} f\right)(t, \omega)=e^{-2 \pi i m t} e^{-2 \pi i n \omega}(Z f)(t, \omega),
$$

$t, \omega \in \Re$. As a special case, if we set $f(x)=\chi_{[0,1)}(x)$, the characteristic function of the interval $[0,1)$, and

$$
\phi_{m, n}(x)=E_{m} T_{n} f(x)=e^{-2 \pi i m x} \chi_{[0,1)}(x-n),
$$

we obtain from (2.4) that

$$
Z\left(\phi_{m, n}\right)(t, \omega)=e^{-2 \pi i m t} e^{-2 \pi i n \omega} \sum_{k \in Z} \chi_{[-k, 1-k)} e^{-2 \pi i k \omega} .
$$

If we restrict $(t, \omega)$ to the unit square $Q$, all the terms in the above summation are zeros except the one that corresponds to $k=0$; hence,

$$
z\left(\phi_{m, n}\right)=E_{m, n}(t, \omega),
$$


where

$$
E_{m, n}(t, \omega)=e^{-2 \pi i m t} e^{-2 \pi i n \omega}=E_{m}(t) E_{n}(\omega)
$$

Therefore, the Zak transform maps, in one-to-one fashion, an orthonormal basis of $L^{2}(\Re),\left\{\phi_{m, n}\right\}_{m, n \in Z}$ onto an orthonormal basis of $L^{2}(Q),\left\{E_{m, n}\right\}_{m, n \in Z}$.

By regarding $f$ in (2.1) as a function of $k$, we may rewrite (2.1) in the form

$$
(Z f)(t, \omega)=\sum_{k \in Z}\left(E_{\omega} T_{-t}\right) f(k)=\sum_{k \in Z} f_{t, \omega}(k),
$$

with $f_{t, \omega}(k)=f(t+k) e^{-2 \pi i k \omega}$.

If we set $g(x)=f_{t, \omega}(x)$, it follows that

$$
\widehat{g}(\gamma)=e^{2 \pi i \omega t} e^{-2 \pi i \gamma t} \widehat{f}(\gamma-\omega)=e^{2 \pi i \omega t} E_{t} T_{\omega} \widehat{f}(\gamma) .
$$

But in view of the Poisson summation formula, we have

$$
\sum_{k \in Z} g(k)=\sum_{k \in Z} \widehat{g}(k)
$$

which, when combined with (2.6) and (2.7), yields

$$
(Z f)(t, \omega)=e^{2 \pi i \omega t}(Z \widehat{f})(-\omega, t),
$$

or equivalently

$$
(Z \widehat{f})(\omega, t)=e^{2 \pi i \omega t}(Z f)(t,-\omega) .
$$

The Zak transform is surprisingly easy to invert. In fact, it is easily seen that

$$
f(t)=\int_{0}^{1}(Z f)(t, \omega) d \omega, \quad t \in \Re
$$

and

$$
\widehat{f}(\omega)=\int_{0}^{1} e^{-2 \pi i \omega t}(Z f)(t, \omega) d t, \quad \omega \in \Re .
$$

Consider the square $Q_{a, b}=[a, a+1] \times[b, b+1]$. Then one can easily verify that

$$
\iint_{Q_{a, b}}(Z f)(t, \omega) \overline{(Z g)}(t, \omega) d t d \omega=\int_{-\infty}^{\infty} f(t) \bar{g}(t) d t .
$$

In particular, when $a=0=b$ we have

$$
\langle Z f, Z g\rangle_{L^{2}(Q)}=\langle f, g\rangle_{L^{2}(\Re)} .
$$

This implies that the Zak transformation is an isometry from $L^{2}(\Re)$ onto $L^{2}(Q)$ and, because of (2.5), it easily follows that it is a unitary transformation from $L^{2}(\Re)$ onto $L^{2}(Q)$.

Now let us introduce the following definitions.

Definition 2.1. Let $\mathcal{P}$ denote the set of all complex-valued functions on $\Re^{2}$ satisfying the following three conditions:

i) $f \in L^{2}(Q)$,

ii) $f(t, \omega+1)=f(t, \omega)$

iii) $f(t+1, \omega)=e^{2 \pi i \omega} f(t, \omega)$. 
Definition 2.2. The convolution, $f \odot g$, of $f, g \in \mathcal{P}$ is defined as

$$
(f \odot g)(t, \omega)=\int_{0}^{1} f(s, \omega) g(t-s, \omega) d s .
$$

Clearly, $Z f \in \mathcal{P}$ if $f \in L^{2}(\Re)$, and it is easy to see that the convolution $\odot$ is well defined. In the notation of [6], the convolution operation symbol $\odot$ is denoted by $*_{t}$, i.e.,

$$
(f \odot g)=\left(f *_{t} g\right)
$$

where the subscript $t$ indicates that the convolution on the right-hand side is taken with respect to the first (translation) parameter. This notation may be confusing since it is inconsistent with the standard convolutional notation

$$
(f * g)(x)=\int_{-\infty}^{\infty} f(y) g(x-y) d y
$$

in which the integral is taken over the whole real line.

Now we state our first lemma, which shows among other things that although the Zak transform of one function is not periodic in the two variables, the product of two Zak transforms indeed is.

Lemma 2.1. Let $f, g \in L^{2}(\Re)$. Then (1)

$$
(Z f)(t, \omega)(\overline{Z g})(t, \omega)=\sum_{m, n \in Z}\left\langle f, E_{m} T_{n} g\right\rangle e^{-2 \pi i m t-2 \pi i n \omega}, \quad t, \omega \in \Re,
$$

(2) if $f * g \in L^{1}(\Re)$, then

$$
Z(f * g)=(Z f) \odot(Z g)
$$

and (3)

$$
Z(f g)=(Z f) \odot_{\omega}(Z g)
$$

where $\odot_{\omega}$ means that $\odot$ is taken over the second (frequency) variable $\omega$.

Proof. (1) Let $H=(Z f)(\overline{Z g})$. Clearly, $H$ is periodic in $\omega$ with period one. Moreover,

$$
\begin{aligned}
H(t+1, \omega) & =\sum_{k, m \in Z} f(t+1+k) \bar{g}(t+1+m) e^{-2 \pi i(k-m) \omega} \\
& =\sum_{k, m \in Z} f(t+k) \bar{g}(t+m) e^{-2 \pi i(k-m) \omega} \\
& =(Z f)(t, \omega)(\overline{Z g})(t, \omega) \\
& =H(t, \omega) .
\end{aligned}
$$

Thus, $H$ is a periodic function with period one in each variable, and consequently it has a Fourier series expansion of the form

$$
H(t, \omega)=\sum_{m, n \in Z} C_{m, n} e^{-2 \pi i m t} e^{-2 \pi i n \omega}
$$

in which

$$
C_{m, n}=\iint_{Q} H(t, \omega) e^{2 \pi i m t} e^{2 \pi i n \omega} d t d \omega
$$


With the aid of (2.4) and (2.10), we have

$$
\begin{aligned}
C_{m, n} & \left.=\iint_{Q}(Z f)(t, \omega) \overline{(Z g}\right)(t, \omega) e^{2 \pi i m t} e^{2 \pi i n \omega} d t d \omega \\
& =\iint_{Q}(Z f)(t, \omega) \overline{Z\left(E_{m} T_{n} g\right)} d t d \omega \\
& =\left\langle Z f, Z\left(E_{m} T_{n} g\right)\right\rangle_{L^{2}(Q)} \\
& =\left\langle f, E_{m} T_{n} g\right\rangle_{L^{2}(\Re)},
\end{aligned}
$$

which, together with (2.14), yields (2.11).

(2) By integrating (2.11) with respect to $t$, we obtain

$$
\int_{0}^{1}(Z f)(t, \omega)(\overline{Z g})(t, \omega) d t=\sum_{n \in Z}\left\langle f, T_{n} g\right\rangle e^{-2 \pi i n \omega}, \quad \omega \in \Re .
$$

For fixed $x$, set $g(y)=\bar{\eta}(x-y)$ in $(2.15)$. Then

$$
\left\langle f, T_{n} g\right\rangle=\int_{-\infty}^{\infty} f(s) \bar{g}(s-n) d s=\int_{-\infty}^{\infty} f(s) \eta(x+n-s) d s=h(x+n)
$$

where $h=f * \eta$. Thus, the right-hand side of $(2.15)$ is exactly $Z h$. On the other hand, since

$$
\begin{aligned}
(\overline{Z g})(t, \omega) & =\sum_{k \in Z} \bar{g}(t+k) e^{2 \pi i k \omega} \\
& =\sum_{k \in Z} \bar{g}(t-k) e^{-2 \pi i k \omega} \\
& =\sum_{k \in Z} \eta(x-t+k) e^{-2 \pi i k \omega} \\
& =(Z \eta)(x-t, \omega),
\end{aligned}
$$

it follows that the left-hand side of (2.15) is just $Z f \odot Z \eta$. Hence, (2.15) can be rewritten in the form $Z f \odot Z \eta=Z(f * \eta)$, which is (2.12).

(3) Formula (2.13) can be derived also from (2.11); however, it is easier to prove it directly. In fact,

$$
\begin{aligned}
(Z f) \odot_{\omega}(Z g) & =\int_{0}^{1}\left(\sum_{k \in Z} f(t+k) e^{-2 \pi i k \gamma}\right)\left(\sum_{k \in Z} g(t+m) e^{-\pi i m(\omega-\gamma)}\right) d \gamma \\
& =\sum_{k, m \in Z} f(t+k) g(t+m) e^{-2 \pi i m \omega} \int_{0}^{1} e^{2 \pi i \gamma(m-k)} d \gamma \\
& =\sum_{k \in Z} f(t+k) g(t+k) e^{-2 \pi i k \omega} \\
& =Z(f g) .
\end{aligned}
$$


We close this section with an example. Let

$$
\delta_{\text {per }}(t)=\sum_{k \in Z} \delta(t-k)
$$

be a periodic extension of the Dirac delta function with period one. It is easy to see that $\delta_{\text {per }}$ has the Fourier series expansion

$$
\delta_{\text {per }}(t)=\sum_{k \in Z} e^{-2 \pi i k t}
$$

Therefore, if $f$ is a periodic function with period one, then we formally have

$$
f(t)=\sum_{m \in Z} c_{m} e^{2 \pi i m t},
$$

and

$$
\begin{aligned}
(Z f)(t, \omega) & =\sum_{k \in Z}\left(\sum_{m \in Z} e^{2 \pi i m(t+k)}\right) e^{-2 \pi i k \omega} \\
& =\sum_{m \in Z} c_{m} e^{2 \pi i m t} \sum_{k \in Z} e^{-2 \pi i k \omega} \\
& =f(t) \delta_{\mathrm{per}}(\omega) .
\end{aligned}
$$

\section{Spaces of functions and generalized functions}

In this section, we introduce spaces of generalized functions that will be needed in the extension of the Zak transform. These spaces, whose elements are called Boehmians, are more general than Schwartz distributions and regular operators [8] and [9]. They were first introduced by J. Mikusiński and P. Mikusiński in [7] as an extension of the notion of regular operators that was developed earlier by $\mathrm{T}$. Boehme in [1].

Unlike the theory of generalized functions developed by Schwartz [10], Gel'fand and Shilov [3], and Zemanian [12], the theory of Boehmians treats generalized functions more as algebraic objects than as elements of the dual space of some testing-function space. Simply put, Boehmians are equivalence classes of "quotients of sequences" constructed from an integral domain of continuous functions as rational numbers are constructed from the integers. For Boehmians, the integral domain operations are addition and a binary operation called convolution. This convolution operation is not necessarily the same as the standard convolution operation denoted earlier by $*$. This flexibility in choosing the convolution operation is what makes the extension of the Zak transform to Boehmians possible.

To make this article self contained, we shall give a brief introduction to Boehmians since they are not very well known.

Boehmians. Let $\mathcal{G}$ be a complex linear space and let $\mathcal{H}$ be a subspace of $\mathcal{G}$. Let $\otimes$ be a binary operation from $\mathcal{G} \times \mathcal{H}$ into $\mathcal{G}$ such that the following conditions are satisfied:

If $\phi, \psi \in \mathcal{H}$, then $\phi \otimes \psi \in \mathcal{H}$ and $\phi \otimes \psi=\psi \otimes \phi$.

If $f \in \mathcal{G}$ and $\phi, \psi \in \mathcal{H}$, then $(f \otimes \phi) \otimes \psi=f \otimes(\phi \otimes \psi)$.

If $f, g \in \mathcal{G}, \phi \in \mathcal{H}$, and $\lambda \in C$, then $(f+g) \otimes \phi=f \otimes \phi+g \otimes \phi$ and $\lambda(f \otimes \phi)=(\lambda f \otimes \phi)$. 
Let $\Delta$ be a family of sequences of elements of $\mathcal{H}$ such that:

$$
\text { if } f \in \mathcal{G},\left\{\phi_{n}\right\} \in \Delta \text {, and } f \otimes \phi_{n}=0 \text { for all } n \in N \text {, then } f=0
$$

(where $N$ is the set of natural numbers), and

$$
\text { if }\left\{\phi_{n}\right\},\left\{\psi_{n}\right\} \in \Delta \text {, then }\left\{\phi_{n} \otimes \psi_{n}\right\} \in \Delta \text {. }
$$

A pair of sequences $\left(f_{n}, \phi_{n}\right)$ is called a quotient of sequences, and will be denoted by $f_{n} / \phi_{n}$, if $f_{n} \in \mathcal{G}$ for $n=1,2, \ldots,\left\{\phi_{n}\right\} \in \Delta$, and $f_{n} \otimes \phi_{m}=f_{m} \otimes \phi_{n}$ for all $m, n \in N$. Two quotients of sequences $f_{n} / \phi_{n}$ and $g_{n} / \psi_{n}$ are equivalent if $f_{n} \otimes \psi_{n}=g_{n} \otimes \phi_{n}$ for every $n \in \boldsymbol{N}$. The equivalence class of $f_{n} / \phi_{n}$ will be denoted by $\left[f_{n} / \phi_{n}\right]$. The space of equivalence classes of quotients of sequences will be denoted by $\mathcal{B}(\mathcal{G}, \mathcal{H}, \otimes, \Delta)$ and its elements will be called Boehmians.

Addition and multiplication by scalars are defined in $\mathcal{B}(\mathcal{G}, \mathcal{H}, \otimes, \Delta)$ as follows:

$$
\begin{gathered}
\lambda\left[f_{n} / \phi_{n}\right]=\left[\lambda f_{n} / \phi_{n}\right] ; \\
{\left[f_{n} / \phi_{n}\right]+\left[g_{n} / \psi_{n}\right]=\left[\left(f_{n} \otimes \psi_{n}+g_{n} \otimes \phi_{n}\right) / \phi_{n} \otimes \psi_{n}\right] .}
\end{gathered}
$$

Clearly, $\mathcal{B}(\mathcal{G}, \mathcal{H}, \otimes, \Delta)$ is a vector space. The mapping $f \rightarrow\left[\left(f \otimes \phi_{n}\right) / \phi_{n}\right]$ is an isomorphism of $\mathcal{G}$ with a subspace of $\mathcal{B}(\mathcal{G}, \mathcal{H}, \otimes, \Delta)$. It is convenient to treat $\mathcal{G}$ as a subspace of $\mathcal{B}(\mathcal{G}, \mathcal{H}, \otimes, \Delta)$.

If $F=\left[f_{n} / \phi_{n}\right] \in \mathcal{B}(\mathcal{G}, \mathcal{H}, \otimes, \Delta)$ and $G=\left[g_{n} / \psi_{n}\right] \in \mathcal{B}(\mathcal{H}, \mathcal{H}, \otimes, \Delta)$, then we can define $F \otimes G$ as follows:

$$
\left.F \otimes G=\left[f_{n} \otimes g_{n}\right) /\left(\psi_{n} \otimes \phi_{n}\right)\right] .
$$

It is easy to see that $F \otimes G \in \mathcal{B}(\mathcal{G}, \mathcal{H}, \otimes, \Delta)$ and that it is an extension of $\otimes$ from $\mathcal{G} \times \mathcal{H}$ onto $\mathcal{B}(\mathcal{G}, \mathcal{H}, \otimes, \Delta) \times \mathcal{B}(\mathcal{H}, \mathcal{H}, \otimes, \Delta)$. Using the identification of $\mathcal{H}$ with a subspace of $\mathcal{B}(\mathcal{H}, \mathcal{H}, \otimes, \Delta)$ we can write

$$
\left[f_{n} / \phi_{n}\right] \otimes \psi=\left[\left(f_{n} \otimes \psi\right) / \phi_{n}\right]
$$

and, in particular, $\left[f_{n} / \phi_{n}\right] \otimes \phi_{k}=f_{k}$.

If $\mathcal{G}$ is equipped with a notion of convergence, then we can define a convergence in $\mathcal{B}$. We say that a sequence of Boehmians $\left\{F_{n}\right\}$ is $\Delta$-convergent to a Boehmian $F$, and we write $F_{n} \stackrel{\Delta}{\rightarrow} F$, if there exits $\left\{\delta_{n}\right\} \in \Delta$ such that $\left(F_{n}-F\right) \otimes \delta_{n} \in \mathcal{G}$ for every $n \in N$ and the sequence $\left\{\left(F_{n}-F\right) \otimes \delta_{n}\right\}$ converges to zero in $\mathcal{G}$. lt can be proved that, under certain conditions, $\mathcal{B}$ equipped with $\Delta$-convergence is a complete quasi-normed space.

It is often convenient to use a second type of convergence: $\delta$-convergence. We say that a sequence of Boehmians $\left\{F_{n}\right\}$ is $\delta$-convergent to a Boehmian $F$, and we write $F_{n} \stackrel{\delta}{\rightarrow} F$, if there exists $\left\{\delta_{n}\right\} \in \Delta$ such that $F_{n} \otimes \delta_{k}, F \otimes \delta_{k} \in \mathcal{G}$, for every $k, n \in N$, and the sequence $\left\{F_{n} \otimes \delta_{k}\right\}$ converges to $F \otimes \delta_{k}$ in $\mathcal{G}$, for every $k \in N$. It can be proved that the sequence $\left\{F_{n}\right\}$ is $\Delta$-convergent to $F$ if and only if every subsequence of $\left\{F_{n}\right\}$ contains a subsequence that is $\delta$-convergent to $F[8]$.

\section{Extension of the Zak transform}

In this section, we discuss briefly Janssen's extension of the Zak transform, then present an alternative approach to the extension problem. We finally conclude this section by comparing our results with his. 

that

Let $\mathcal{S}$ be the space of all entire functions $f$ for which there exist $A, B, M>0$ such

$$
|f(x+i y)| \leq M e^{\left(-\pi A x^{2}+\pi B y^{2}\right)}, \quad x, y \in \Re .
$$

The topology of $\mathcal{S}$ is defined as follows: a sequence $\left\{f_{n}\right\}_{n=0}^{\infty}$ in $\mathcal{S}$ converges to zero in $\mathcal{S}$ if there exist $A, B, M>0$ such that (4.1) holds for all $f_{n}$ and $f_{n} \rightarrow 0$ pointwise as $n \rightarrow \infty$. The dual space of $\mathcal{S}$ will be denoted by $\mathcal{S}^{*}$ and the action of $F \in \mathcal{S}^{*}$ on $f \in \mathcal{S}$ will be denoted by $\langle F, f\rangle$. The Schwartz space of $C^{\infty}$ rapidly decreasing functions will be denoted by $\boldsymbol{S}$ and its dual, the space of tempered distributions, will be denoted by $\boldsymbol{S}^{*}$.

Following the notation of Gel'fand and Shilov [3, p. 179], we define the space $S_{\alpha}^{\beta}$ as the space of all infinitely differentiable functions $f(x)$ satisfying

$$
\left|x^{k} f^{(q)}(x)\right| \leq C A^{k} B^{q} k^{k \alpha} q^{q \beta}, \quad k, q=0,1,2, \ldots
$$

for some positive constants $A, B$, and $C$. It is known that every function $f \in S_{\alpha}^{\beta}$ satisfies the estimate

$$
\left|f^{(q)}(x)\right| \leq C B^{q} q^{q \beta} \exp \left(-a|x|^{1 / \alpha}\right)
$$

for some $a>0$. If $0 \leq \beta<1$, then $f$ can be continued analytically to the complex $z$-plane, $z=x+i y$, and, in addition, $f$ satisfies the estimate

$$
\left|x^{k} f(x+i y)\right| \leq C A^{k} k^{k \alpha} \exp \left(b|y|^{1 /(1-\beta)}\right) .
$$

Janssen's space $\mathcal{S}$ can be identified with the space $S_{1 / 2}^{1 / 2}$. To show how large the space $S^{*}$ is, we note that any measurable function $F(x)$ for which $\int_{-\infty}^{\infty}|F(x)| e^{-\varepsilon x^{2}} d x<\infty$ for all $\varepsilon>0$ gives rise to an element in $\mathcal{S}^{*}$ by putting

$$
\langle F, f\rangle=\int_{-\infty}^{\infty} F(x) \overline{f(x)} d x, \quad f \in \mathcal{S},
$$

which exists in view of (4.1). It is easily seen that this defines a continuous functional on $\mathcal{S}$.

To extend the Zak transform to larger spaces than $L^{2}(\Re)$, Janssen used the notion of a tensor product of generalized functions. He defined the tensor product $\otimes$ of $F_{1}, F_{2} \in \mathcal{S}^{*}$ as

$$
\left\langle F_{1} \otimes F_{2}, f_{1} \otimes f_{2}\right\rangle=\left\langle F_{1}, f_{1}\right\rangle\left\langle F_{2}, f_{2}\right\rangle,
$$

for $f_{1}, f_{2} \in \mathcal{S}$. In particular, if $F_{2}=1$, we have

$$
\left\langle F_{1} \otimes 1, f_{1} \otimes f_{2}\right\rangle=\left\langle F_{1}, f_{1}\right\rangle \int_{-\infty}^{\infty} f_{2}(x) d x .
$$

The Zak transform of $F \in \mathcal{S}^{*}$ is defined as

$$
(Z F)(t, \omega)=\sum_{k \in Z} T_{-k}^{1} E_{k}^{2}(F \otimes 1)=\sum_{k \in Z}\left(T_{-k} F \otimes E_{k} 1\right)
$$

where the superscripts 1,2 indicate that the operator is applied to the first and second element in the tensor product, respectively. The following are shown in [5]. 
1) Definition (4.3) makes sense, since for $f_{1}, f_{2} \in \mathcal{S}$, the series

$$
\sum_{k \in Z}\left\langle T_{-k} F \otimes E_{k} 1, f_{1} \otimes f_{2}\right\rangle=\sum_{k \in Z}\left\langle T_{-k} F, f_{1}\right\rangle \overline{\widehat{f}_{2}}(k)
$$

converges absolutely.

2) The Zak transformation is a continuous linear transformation from $\mathcal{S}^{*}$ into $(\mathcal{S} \times \mathcal{S})^{*}=\left(\mathcal{S}^{2}\right)^{*}$, and also from $\boldsymbol{S}^{*}$ into $(\boldsymbol{S})^{*}$.

3) If $F$ belongs to $\mathcal{S}$ or $\boldsymbol{S}$, then $Z F$ agrees with the standard definition (2.1).

4) For $F$ in $\mathcal{S}^{*}$ or $\boldsymbol{S}^{*}$ and $f$ in $\mathcal{S}$ or $\boldsymbol{S}$, respectively, we have an analogue of (2.11),

$$
\sum_{m, n \in Z}\left\langle F, E_{m} T_{n} f\right\rangle e^{-2 \pi i m t} e^{-2 \pi i n \omega}=(Z F)(t, \omega) \overline{(Z f)}(t, \omega)
$$

where the series converges in the sense of $\left(\mathcal{S}^{2}\right)^{*}$ or $\left(\boldsymbol{S}^{2}\right)^{*}$.

The Zak transforms of $\mathcal{S}, \boldsymbol{S}, \mathcal{S}^{*}$, and $\boldsymbol{S}^{*}$ can be characterized as follows: $Z(\mathcal{S})$ is equal to the set of all entire functions $f$ of two variables satisfying

$$
f(z+1, w)=e^{-2 \pi i w} f(z, w), \quad f(z, w+1)=f(z, w), \quad \text { for all } z, \omega \in C,
$$

and such that there exist constants $A, B, M>0$ with

$$
|f(x+i y, u+i v)| \leq M \exp \left(2 \pi x v+\pi A y^{2}+\pi B v^{2}\right)
$$

while

$$
Z\left(\mathcal{S}^{*}\right)=\left\{F \in\left(\mathcal{S}^{2}\right)^{*}: T_{-1}^{1} F=E_{1}^{2} F \quad \text { and } \quad T_{-1}^{2} F=F\right\} .
$$

On the other hand, $Z(\boldsymbol{S})$ is equal to the set of all functions of two variables $f(z, w)$ that are $C^{\infty}$ in both variables and satisfy

$$
f(z+1, w)=e^{-2 \pi i w} f(z, w), \quad f(z, w+1)=f(z, w) \quad \text { for all } z, w,
$$

while

$$
Z\left(\boldsymbol{S}^{*}\right)=\left\{F \in\left(\boldsymbol{S}^{2}\right)^{*}: T_{-1}^{1} F=E_{1}^{2} F \quad \text { and } \quad T_{-1}^{2} F=F\right\} .
$$

Let us denote $Z(\boldsymbol{S})$ by $\widetilde{\mathcal{P}}$. Clearly, $\widetilde{\mathcal{P}} \subset \mathcal{P}$; see Definition 2.1.

Definition 4.1. A sequence $\left\{\delta_{n}\right\}_{n=1}^{\infty}$ of real-valued functions with $\delta_{n} \in S$ is called a delta sequence if
i) $\int_{-\infty}^{\infty} \delta_{n}(x) d x=1$ for all $n$,
ii) $\int_{-\infty}^{\infty}\left|\delta_{n}(x)\right| d x<M$ for some $M>0$ and all $n$,
iii) $\lim _{n \rightarrow \infty} \int_{|x| \geq \varepsilon}\left|\delta_{n}(x)\right| d x=0$ for all $\varepsilon>0$.

The collection of all such delta sequences will be denoted by $\Delta_{S}$. The reader can easily verify that for $\mathcal{G}=L^{2}(\Re), \mathcal{H}=S, \otimes=*$ and $\Delta=\Delta_{S}$, conditions (3.1)-(3.5) are satisfied. The corresponding space of Boehmians will be denoted by $\mathcal{B}_{L^{2}}$, i.e.,

$$
\mathcal{B}_{L^{2}}=\mathcal{B}\left(L^{2}(\Re), S, *, \Delta_{S}\right) .
$$

Elements of $\mathcal{B}_{L^{2}}$ are called square integrable Boehmians. 
Lemma 4.1. Let $\Delta_{Z}=\left\{\left\{Z \phi_{n}\right\}:\left\{\phi_{n}\right\} \in \Delta_{S}\right\}$. Then $\mathcal{B}_{Z}=\mathcal{B}\left(\mathcal{P}, \widetilde{\mathcal{P}}, \odot, \Delta_{Z}\right)$ is a class of Boehmians.

Proof. The proof is straightforward; we only need to use (2.12) to verify that (3.1)(3.5) hold.

Theorem 4.2. If $F=\left[f_{n} / \phi_{n}\right] \in \mathcal{B}_{L^{2}}$, then $\left[\left(Z f_{n}\right) /\left(Z \phi_{n}\right)\right] \in \mathcal{B}_{Z}$.

Proof. Since $f_{n} \in L^{2}(\Re), \phi_{n} \in S$, it follows that $\left(Z f_{n}\right) \in \mathcal{P}$ and $\left(Z \phi_{n}\right) \in \widetilde{\mathcal{P}}$. Now it suffices to show that

$$
\left(Z f_{n}\right) \odot\left(Z \phi_{m}\right)=\left(Z f_{m}\right) \odot\left(Z \phi_{n}\right)
$$

for all $m$ and $n$. But this is an immediate consequence of (2.12) since

$$
\left(Z f_{n}\right) \odot\left(Z \phi_{m}\right)=Z\left(f_{n} * \phi_{m}\right)=Z\left(f_{m} * \phi_{n}\right)=\left(Z f_{m}\right) \odot\left(Z \phi_{n}\right) .
$$

Now we are able to present our extension of the Zak transform.

Definition 4.2. The Zak transform of $F=\left[f_{n} / \phi_{n}\right] \in \mathcal{B}_{L^{2}}$ is defined as the Boehmian $\left[\left(Z f_{n}\right) /\left(Z \phi_{n}\right)\right] \in \mathcal{B}_{Z}$ and will be denoted by $Z F$.

For this definition to make sense, we must show that the definition is independent of the representation of $F$. But this is easy, since if $F=\left[f_{n} / \phi_{n}\right]=\left[g_{m} / \psi_{m}\right]$, then

$$
\left(Z f_{n}\right) \odot\left(Z \psi_{m}\right)=Z\left(f_{n} * \psi_{m}\right)=Z\left(g_{m} * \phi_{n}\right)=Z\left(g_{m}\right) \odot Z\left(\phi_{n}\right) .
$$

From (2.9) and definition 4.2, it is easy to prove

Theorem 4.3 (Inversion formula). Let $F \in \mathcal{B}_{L^{2}}$ and $Z F=\left[g_{m} / \gamma_{n}\right]$. Then

$$
F=\left[\left(Z^{-1} g_{n}\right) /\left(Z^{-1} \gamma_{n}\right)\right]
$$

Note that the Zak transform is an algebraic isomorphism of $\mathcal{B}_{L^{2}}$ and $\mathcal{B}_{Z}$. We are going to show that it is also a topological isomorphism.

Recall that a sequence of Boehmians $F_{n} \in \mathcal{B}_{L^{2}}$ is $\Delta$-convergent to a Boehmian $F \in \mathcal{B}_{L^{2}}$, denoted by $F_{n} \stackrel{\Delta}{\rightarrow} F$, if there exists a delta sequence $\left\{\phi_{n}\right\} \in \Delta_{S}$ such that $\left(F_{n}-F\right) * \phi_{n} \in L^{2}(\Re)$ for all $n \in N$, and $\left(F_{n}-F\right) * \phi_{n} \rightarrow 0$ in $L^{2}(\Re)$, as $n \rightarrow \infty$.

A sequence of Boehmians $F_{n} \in \mathcal{B}_{Z}$ is $\Delta$-convergent to a Boehmian $F \in \mathcal{B}_{Z}$, denoted by $F_{n} \stackrel{\Delta}{\rightarrow} F$, if there exists a delta sequence $\left\{\phi_{n}\right\} \in \Delta_{Z}$ such that $\left(F_{n}-F\right) \odot \phi_{n} \in \mathcal{P}$ for all $n \in N$ and $\int_{Q}\left|\left(F_{n}-F\right) \odot \phi_{n}\right|^{2} \rightarrow 0$, as $n \rightarrow \infty$.

Theorem 4.4. $F_{n} \stackrel{\Delta}{\rightarrow} F$ in $\mathcal{B}_{L^{2}}$ if and only if $Z F_{n} \stackrel{\Delta}{\rightarrow} Z F$ in $\mathcal{B}_{Z}$.

Proof. It suffices to prove the theorem for $F=0$. So let $F_{n} \triangleq 0$ in $\mathcal{B}_{L^{2}}$ and let $\left\{\phi_{n}\right\} \in \Delta_{S}$ be such that $f_{n}=F_{n} * \phi_{n} \in L^{2}(\Re)$ for all $n$ and $f_{n} \rightarrow 0$ in $L^{2}(\Re)$. Then, since $\left\{Z \phi_{n}\right\} \in \Delta_{Z}$, we have

$$
Z F_{n} \odot Z \phi_{n}=Z\left(F_{n} * \phi_{n}\right)=Z\left(f_{n}\right)
$$

which implies that $\left(Z F_{n} \odot Z \phi_{n}\right) \in \mathcal{P}$. Moreover, by (2.10)

$$
\left\|\left(Z F_{n} \odot Z \phi_{n}\right)\right\|_{L^{2}(Q)}=\left\|Z f_{n}\right\|_{L^{2}(Q)}=\left\|f_{n}\right\|_{L^{2}(\Re)} \rightarrow 0, \quad \text { as } n \rightarrow \infty .
$$

Thus $Z F_{n} \stackrel{\Delta}{\rightarrow} 0$ in $\mathcal{B}_{Z}$. The proof in the other direction is similar.

Corollary 4.5. The Zak transform is a topological isomorphism of $\mathcal{B}_{L^{2}}$ onto $\mathcal{B}_{Z}$. 
Our extension of the Zak transform appears to be simpler and more natural than Janssen's; now we show that it is actually more general. We do that by constructing a Boehmian $F \in \mathcal{B}_{L^{2}}$ that is not an element of $\mathcal{S}^{*}$.

Choose $\phi \in S$ such that $\int_{-\infty}^{\infty} \phi(x) d x=1$ and

$$
\left|x^{q} \phi^{(k)}(x)\right| \leq C_{q} A^{k} k^{k / 6} \text { for all } q, k=0,1,2, \ldots
$$

where $A$ and $C_{q}$ are constants with $C_{q}$ being dependent on $q$ but not $k$. Here we adopt the notation that $0^{0}=1$. Condition (4.4) is possible in view of (4.2) and the fact that $S=\bigcup_{0<\alpha}\left(\bigcup_{0<\beta} S_{\alpha}^{\beta}\right)$.

Define

$$
\phi_{n}(x)=n \phi(n x), \quad n=1,2, \ldots,
$$

and

$$
f_{n}(x)=\sum_{k=0}^{\infty} \frac{1}{k^{k / 3}} \phi_{n}^{(k)}(x) .
$$

Note that $\left\{\phi_{n}\right\}_{n=1}^{\infty} \in \Delta_{S}$, and that for every $q$,

$$
\begin{aligned}
\sup _{x \in \Re}\left|x^{q} \phi_{n}^{(k)}(x)\right| & =\sup _{x \in \Re}\left|x^{q} n^{k+1} \phi^{(k)}(n x)\right| \\
& =\sup _{x \in \Re}\left|n^{k+1-q}(n x)^{q} \phi^{(k)}(n x)\right| \\
& \leq n^{k+1-q} C_{q} A^{k} k^{k / 6} .
\end{aligned}
$$

Hence, the series defining $f_{n}$ converges to an element in $\boldsymbol{S}$, in particular, $f_{n} \in L^{2}(\Re)$. Moreover, it can be easily verified that

$$
f_{n} * \phi_{m}=f_{m} * \phi_{n}
$$

which shows that $F=\left[f_{n} / \phi_{n}\right] \in \mathcal{B}_{L^{2}}$. Choose $\psi \in \mathcal{S}$ such that

$$
\psi^{(k)}(0)=C_{q} B^{k} k^{k / 2}, \quad k=0,1, \ldots,
$$

which is again possible in view of (4.2). Consider

$$
\widetilde{F}(x)=\sum_{k=1}^{\infty} \frac{1}{k^{k / 3}} \delta^{(k)}(x) .
$$

It is readily seen that $\widetilde{F} * \phi_{n}=f_{n}$ for $n=1,2, \ldots$, hence $\widetilde{F}=F \in \mathcal{B}_{L^{2}}$. But (4.5) implies that the series

$$
\langle F, \psi\rangle=\sum_{k=1}^{\infty} \frac{1}{k^{k / 3}}(-1)^{k} \psi^{(k)}(0)
$$

diverges, and thus $F \notin \mathcal{S}^{*}$. 


\section{References}

1. T. K. Boehme, The support of Mikusiński operators, Trans. Amer. Math. Soc. 176 (1973), 319334.

2. I. Daubechies, Ten Lectures on Wavelets, CBMS-NSF, Regional Conferences Series in Applied Math., SIAM Publication, Philadelphia, 1992.

3. I. M. Gel'fand and G. E. Shilov, Generalized Functions, Vol. II, Academic Press, New York, 1964.

4. C. E. Heil and D. F. Walnut, Continuous and discrete wavelet transforms, SIAM Rev. 31 (1989), $628-666$.

5. A. J. E. M. Janssen, Bargmann transform, Zak transform, and coherent states, J. Math. Phys., 23 (1982), 720-731.

6. - The Zak transform: a signal transform for sampled time-continuous signals, Philips J. Res., 43 (1988), 23-69.

7. J. Mikusiński and P. Mikusińki, Quotients de suites et leurs applications dans I'analyse fonctionnelle, Comptes Rendus, Serie I 293 (1981), 463-464.

8. P. Mikusiński, Convergence of Boehmians, Japan J. of Math., 9 (1983), 159-179.

9. __ Boehmians and generalized functions, Acta Math. Hungarica 51 (1988), 271-281.

10. L. Schwartz, Theorie des Distributions, Hermann Publ. Paris, 1950-1951.

11. J. Zak, Finite translations in solid state physics, Phys. Rev. Lett. 19 (1967), 1385-1397.

12. A. H. Zemanian, Generalized Integral Transformations, Dover Publ., New York, 1987.

Department of Mathematics, University of Central Florida, Orlando, Fl 32816, U.S.A. 